\title{
Person Detection and Following Quadcopter System Based on the Color Information
}

\author{
Noriyuki Kawarazaki*, Masato Koizumi and Tadashi Yoshidome \\ Kanagawa Institute of Technology, Shimo-Ogino 1030 Atsugi, Kanagawa 243-0292, Japan \\ ${ }^{*}$ Corresponding author
}

\begin{abstract}
This paper discussed a person detection and following quadcopter system based on the color information. This quadcopter system detects the target person based on the color of the person's jacket and follows the person to keep the constant distance. The application of our quadcopter is the indoor navigation system for a visitor. In our system the quadroter is controlled by computer program via Wi-Fi. We use the simple and conventional object detection algorithm of the image processing. In order for the quadcopter to follow the target person, the system calculates the speeds of four rotors based on the position of the person. The advantage of the quadcopter can move up and down the stair. The effectiveness of our system is demonstrated by several performance tests.
\end{abstract}

Keywords-quadcopter; person detection and following; image processing; navigation system

\section{INTRODUCTION}

The development of robots has been important to industrial production, including that in factories. Intelligent robots are expected to work cooperatively with people in daily life and in medical treatment and welfare. It is necessary for the intelligent mobile robot to detect and follow the parson to support human works. For example, an intelligent cargo transportation robot carries the luggage in place of a porter at the airport. The mobile cleaning robot to track the sound source using the three microphones has been developed [1]. The mobile robot system for tracking and following moving people using an omnidirectional camera and a laser was developed [2]. A human tracking system for an autonomous mobile robot using neural-based motion detectors has been proposed [3]. This system uses motion sensors based on the motion sensitive neurons found in the medial temporal area of the primate brain. The target tracking system for a mobile robot under varying illumination using stereo camera has been proposed [4]. The localization of mobile robot using images by distributed intelligent networked devices has been proposed [5]. This method is used the Kalman filtering scheme to estimate the location of moving robot. The mobile robot system that has functions of human following and returning to the starting location autonomously while avoiding obstacles using LRF(Laser Range Finder) and camera was developed [6]. However, one of the problems is that the mobile robot can't move the non-leveling of ground and the stairs.

On the other hand, the many kinds of flying robots called drones begin to de developed in recent years. The flying robots have many potential applications in indoor environments. The lightweight flying robot for achieving indoor autonomous flight using four infrared sensors has been developed [7]. The visionbased line tracking algorithm for an indoor micro quadrotor has been proposed [8]. The system that enables a monocular-vision based quadrotor to automatically recover from any unknown was developed [9].

This paper presented a person detection and following quadcopter system based on the color information. The application of our quadcopter is the indoor navigation system for a visitor. The quadcopter can detect and follow the target person based on color image from the camera. The goal of our system is that the quadcopter can follow the walking person in the indoor environment. The advantage of the quadcopter can move up and down the stair. The effectiveness of our system is clarified by several performance tests.

\section{HUMAN FOLLOWING QUADCOPTER}

We developed a person detection and following quadcopter system based on the color information. The outline of the person detection and following quadcopetr system is shown in Figure I. This quadcopter system detects the target person based on the color of the person's jacket and follows the person to keep the constant distance. This quadcopter system is composed of the manufactured quadcopter with color camera (AR.Drone 2.0, Parrot [10]), and control PC (Let's note, Panasonic). The overview and specification of the quadroter are shown in Figure II and Table I respectively. The AR.Drone is a remote controlled flying quadcopter which has four rotors. In our system the quadcopter is controlled by computer program via Wi-Fi. As shown in Figure II, the quadroter has a RGB camera at the front part. The color image of the front camera is sent to the control PC via Wi-Fi. The system detects the target person in the color image and controls the quadroter to follow the person. We use the image processing library OpenCV [11] for the implementation of the system.

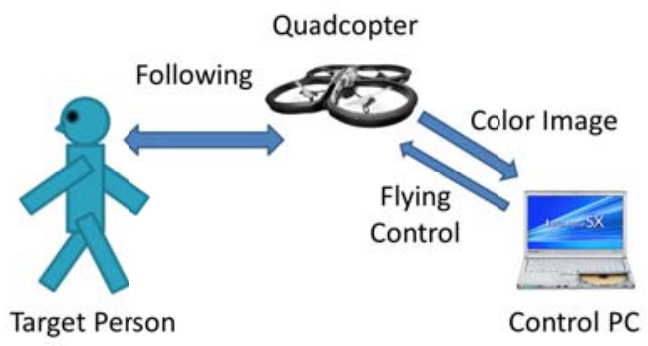

FIGURE I. PERSON DETECTION AND FOLLOWING QUADCOPTER SYSTEM 


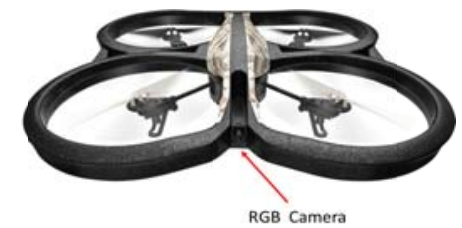

FIGURE II. OVERVIEW OF QUADCOPTER

TABLE I. SPECIFICATIONS OF QUADCOPTER

\begin{tabular}{|c|c|}
\hline Parameters & Specifications \\
\hline Size & $517 \times 517 \times 127[\mathrm{~mm}]$ \\
\hline Weight & $420[\mathrm{~g}]$ \\
\hline Body Material & Carbon Fiber \\
\hline Maximum Flight Speed & $11.1[\mathrm{~m} / \mathrm{sec}]$ \\
\hline Maximum Flying Attitude & $100[\mathrm{~m}]$ \\
\hline Maximum Flight Time & $12[\mathrm{~min}]$ \\
\hline Payload capacity & $100[\mathrm{~g}]$ \\
\hline Front Camera & $640 \times 480[\mathrm{pix}]$ \\
\hline OS & Linux \\
\hline CPU & ARM9 $(468 \mathrm{MHz})$ \\
\hline Flight Sensors & Acceleration, Jyro \\
\hline
\end{tabular}

\section{ERSON DETECTION BASED ON THE COLOR INFORMATION}

We suppose that the target person wears the monochrome jacket, since the system detects the target person based on the jacket's color. The system detects the target person based on the jacket's color. The general flow of the target detection based on the image processing is shown in Figure III. We use the simple and conventional object detection algorithm of image processing to detect the target person.

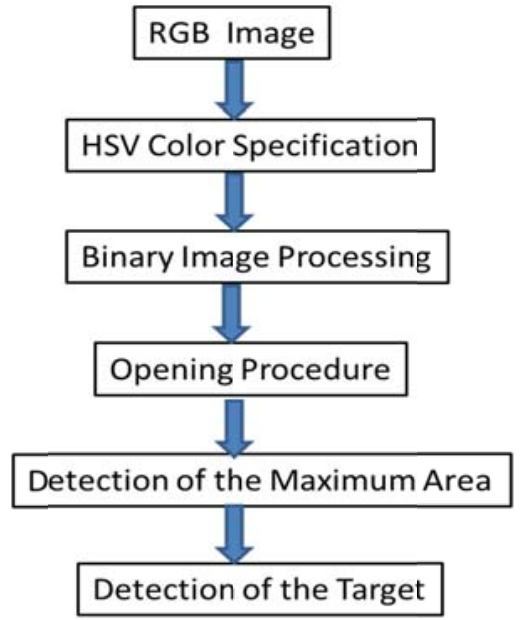

FIGURE III. GENERAL FLOW OF THE TARGET DETECTION

The quadcopter sends the RGB image from the front camera. The target person is detected based on the pixel values of the jacket's color. The color image is digitized as RGB (Red, Green and Blue) pixel value, so that each element of RGB is several levels of brightness. The original RGB image is shown in Figure IV(a). However, the RGB value is apt to be influenced by the light. Therefore, we use the HLS (hue, saturation, and value) color specification system in order to detect the person's jacket accurately. The each elements of HLS color specification system are calculated based on the RGB pixel value [12]. The example of the HSV color specification is shown in Figure IV(b). In order to detect the target person in the color image, we transform color image to the binary image based on the threshold values of HSL color specification system. We define the threshold values of HSL color specification system so as to detect the person's jacket accurately. The binary image is shown in Figure IV(c). In order to remove pixel noise from the binary image, the opening procedure is executed at the binary image. The opening procedure is the combination of an erosion followed by a dilation. In our system, an erosion and a dilation were executed ten times respectively. As shown in Figure IV(d), the pixel noises were removed in the binary image. After the detection of the object regions in the binary image, the system calculates the region's areas. We suppose that the region with the maximum area is the target object. The Figure IV(e) shows the region with the maximum area. Finally, the system detects the target object in the color image (see Figure IV(f)).

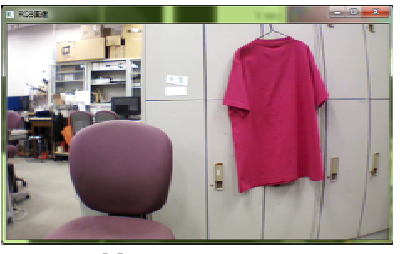

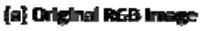

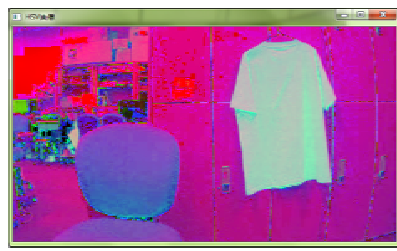

(b) HSV Calur Spedification

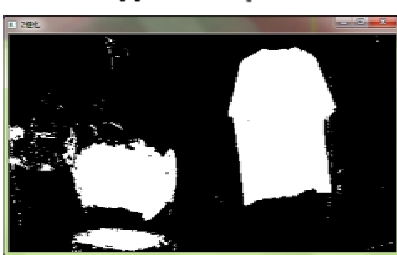

(c) Dinary image Processing

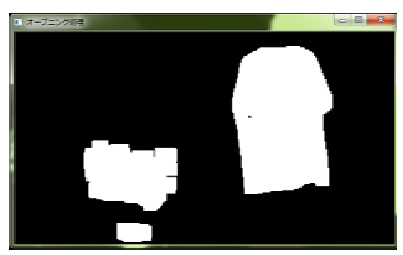

(d) Openine Procture

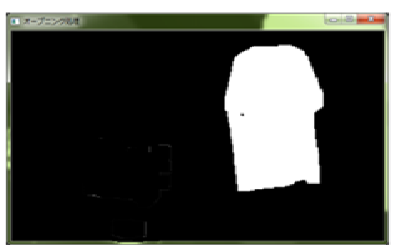

(el Detection of the Madinum Anea

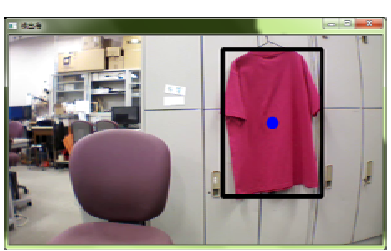

(f) Dedection of the Taret
FIGURE IV. EXAMPLE OF THE TARGET DETECTION

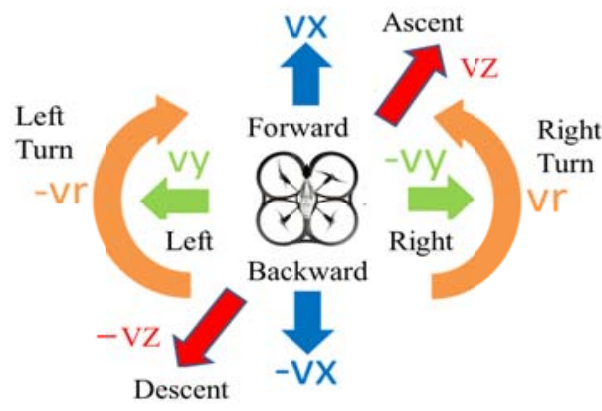

FIGURE V. MOVEMENTS OF QUADCOPTER 


\section{Person Following Control of the QuAdCOPTER}

The mechanical structure of quadcopter comprises four rotors with motors. Each pair of opposite rotors is turning the same way. One pair is turning clockwise and the other anticlockwise. As shown in Figure V, quadcopter moves eight kinds of motion (Forward, Backward, Right, Left, Right Turn, Left Turn, Ascent, Descent). Each motion of the quadcopter is executed by changing the speeds of four rotors. For example, the system varies the front rotor speed the quadcopter goes forward. If the system adds the four rotor speeds in the same way, the quadcopter moves upward.

In order for the quadcopter to follow the target person, the system calculates the speeds of four rotors based on the position of the person. The position of the person is recognized based on the variance of the initial situation of the target person At first, the target person stands at the position approximately $2 \mathrm{~m}$ away from the quadcopter. Then the quadcopter lifts up at the $1.5 \mathrm{~m}$ from the floor. At that position, system captures the color image and detects the person's jacket using the image processing. The system defines the standard frame which is a surrounding rectangle of the person's jacket (see Figure VI). When the target person starts to walk, the system captures the color image and detects the person's jacket. The system defines the target frame which is surrounding rectangle of the person's jacket. The system calculates the differences between the standard frame and the target frame. The motion command according to the four rotor speeds is determined based on this difference. The control PC sends the motion command to the quadcopter to follow the target person.

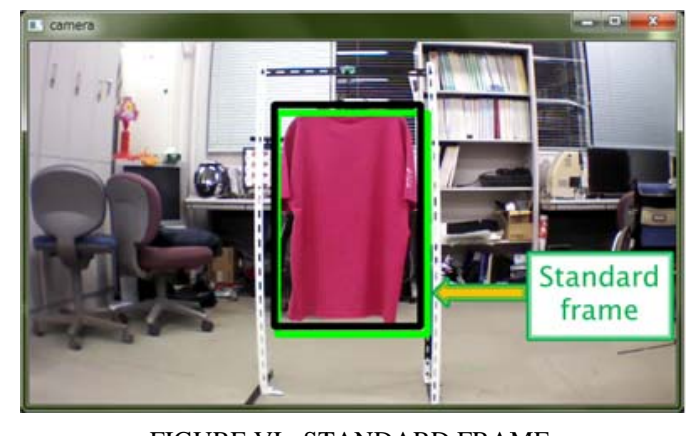

FIGURE VI. STANDARD FRAME

\section{Forward or Backward Motion}

The motion command of the forward or backward of the quadrotor is determined based on the difference between the initial frame size and the target frame size. As shown in Figure VII(a), when the quadcopter leaves from the target person, the target frame size becomes small comparing with the initial frame size. Conversely, when the quadcopter approaches to the target person, the target frame size becomes large (see Figure VII(b)). Then the rotor speed of the forward or backward motion is calculated based on the difference between widths of the two frames.

\section{Ascent or Descent Motion}

The motion command of the ascent or descent of the quadcopter is determined based on the center position of the target frame. As shown in Figure VIII, we define the three regions (ascent, initial and descent) in the image. When the center point of the target frame exists in the domain of a onethird upper part of the image (ascent region), the quadcopter is flying high position comparing with the initial position. The quadrotor is controlled so that the center position of the target frame enters into the initial region. The rotor speed of the ascent or descent motion is calculated based on the distance from the target frame center position to the initial region center position.

\section{$\underline{\text { Right or Left Motion }}$}

The motion command of the right or left of the quadcopter is determined based on the center position of the target frame. As shown in Figure IX, the quadrotor is controlled so that the center position of the target frame coincides with the center position of the initial frame. The rotor speed of the right or left motion is calculated based on the distance from the target frame center position to the initial region center position.

\section{Right Turn or Left Turn Motion}

The motion command of the right turn or left turn of the quadcopter is determined based on the center position of the target frame. The quadcopter is controlled so that the center position of the target frame coincides with the center position of the initial frame.

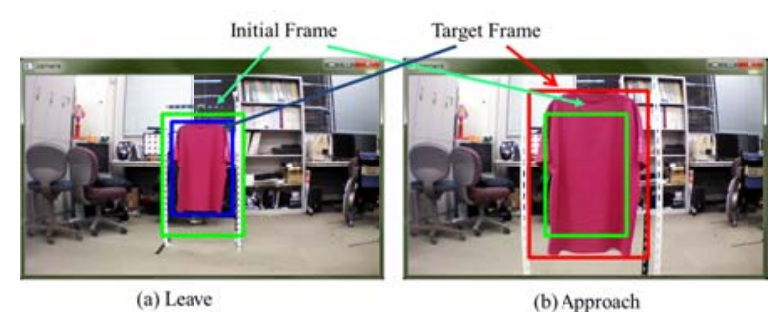

FIGURE VII. SITUATIONS OF LEAVE OR APPROACH MOTION

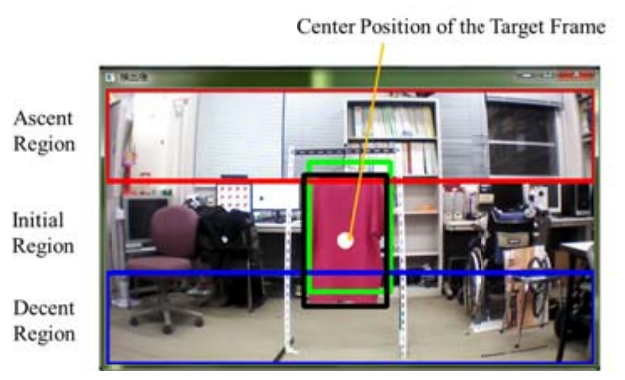

FIGURE VIII. SITUATIONS OF ASCENT OR DESCENT MOTION

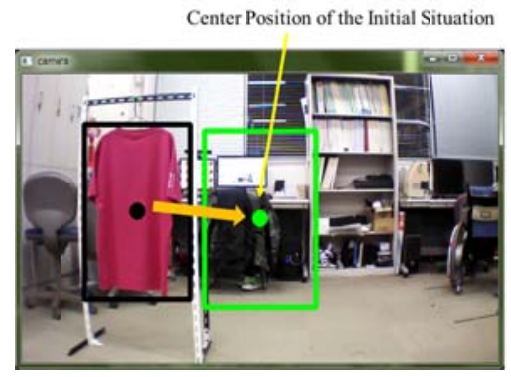

FIGURE IX. SITUATIONS OF RIGHT (RIGHT TURN) OR LEFT (LEFT TURN) MOTION 


\section{EXPERIMENTAL RESULTS}

We conducted two performance tests to clarify the effectiveness of our system.

\section{Performance Test1}

The quadcopter follows the target person who walks straight the flat corridor at $20 \mathrm{~m}$. The initial situation is that the quadcopter is approximately $2 \mathrm{~m}$ away from the target person. The three subjects (all men in twenties) participated in the test1. The each subject was tested 5 times. The situation of the performance test 1 is shown in Figure X. The figure XI shows the last state of the position relation between the quadcopter and the target person. As shown in Figure XI, the quadcopter followed the target person to keep the distance $2 \mathrm{~m}$ from the person. The success rate of the performance test 1 is $100 \%$.

\section{Performance Test2}

The quadcopter follows the target person who goes up 11 steps of stairs. The initial situation is that the quadcopter is approximately $2 \mathrm{~m}$ away from the target person. The three subjects (all men in twenties) participated in the test 2. The each subject was tested 5 times. The situation of the performance test2 is shown in Figure XII. The success rate of the performance test 2 is $80 \%$. The reason of the failure was that the system didn't recognize the target frame accurately under the influence of a change of the light.

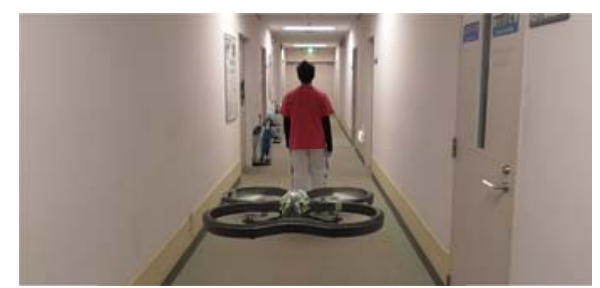

FIGURE X. SITUATION OF PERFORMNCE TEST1

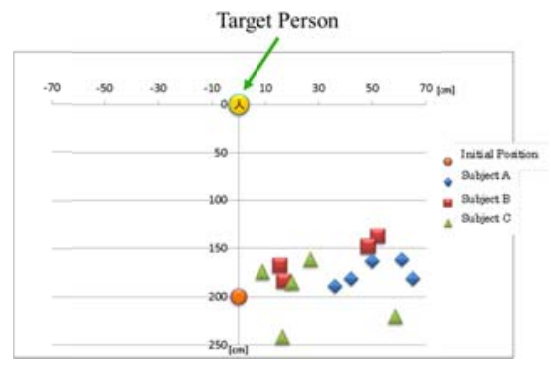

FIGURE XI. LAST STATE OF PERFORMNCE TEST1

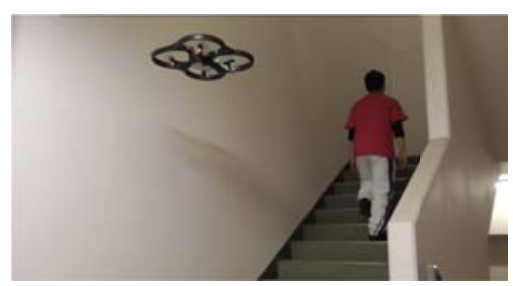

FIGURE XII. SITUATION OF PERFORMNCE TEST 2

\section{CONCLUSIONS}

In this paper, we developed a person detection and following quadcopter system based on the color information. This quadcopter system detects the target person based on the color of the person's jacket and follows the person to keep the constant distance. In order for the quadcopter to follow the target person, the system calculates the speeds of four rotors based on the position of the person. The position of the person is recognized based on the variance of the initial situation of the target person. We conducted performance tests to clarify the effectiveness of the system. The success rate of the performance test 1 was $100 \%$ and test 2 was $80 \%$ respectively. In our future work, we intend to explore further the practicality of the system by various experimental scenarios.

\section{REFERENCES}

[1] Jongho Han and Taeseok Jin, "Sound Source based Mobile Robot Control for Human Following in a Networked Intelligent Space”, International Journal of Control and Automation, Vol. 8, No. 7,pp.67-74, 2015

[2] M. Kobilarov, G. Sukhatme, J. Hyams and P. Batavia, "People tracking and following with mobile robot using an omnidirectional camera and a laser”. Proc. of the 2006 IEEE Int. Cinf. on Robotics and Automation, p557-562, 2006.

[3] J. A. Perrone, T. Voyle, M. E. Jefferies, "Toward a Human Tracking System for a Mobile Robot Using Neural-Based motion Detectors", Image and Vision Computing NZ, p.24-28. 2003.

[4] Yuzuka Isobe, Gakuto Masuyama, and Kazunori Umeda, "Human Following with a Mobile Robot Based on Combination of Disparity and Color Images”, Proceeding of the 10th France - Japan Congress, 8th Europe - Asia Congress on Mecatronics, pp.84-88,2014.

[5] T. Jin, J. Lee and H. Hashimoto, "Position Control of Mobile Robot for Human-Following in Intelligent Space with Distributed Sensors", International Journal of Control, Automation, and Systems, vol.4, no.2, p.204-216,2006.

[6] Takahito Shimizu, Masashi Awai, Atsushi Yamashita and Toru Kaneko, "Mobile Robot System Realizing Human Following and Autonomous Returning Using Laser Range Finder and Camera”, Proceedings of the 18th Korea-Japan Joint Workshop on Frontiers of Computer Vision (FCV2012), pp.97-102, 2012.

[7] Daisuke Iwakura and Kenzo Nonami, "Indoor Localozation of Flying Robot by Means of Infrared Sensors”, Journal of Robotics and Mechatronics, Vol.25, No.1, pp.201-210, 2013

[8] Han Sol Kim, Andrew Hong and Jin Bae Park, "Vision-based Line Tracking for an Indoor Micro Quadrotor”, Journal of International Council on Electrical Engineering, Vol.3, No.3, pp.245-249, 2013

[9] Matthias Faessler, Flavio Fontana, Christian Forster and Davide Scaramuzza, "Automatic Re-Initialization and Failure Recovery for Aggressive Flight with a Monocular Vision-Based Quadrotor", Proceedings of the 2015 IEEE International Conference on Robotics and Automation, pp.1722-1729, 2015.

[10] http://ardrone2.parrot.com/

[11] http://opencv.jp/opencv-2.1/c/index.html

[12] John C. Russ : “The Image Processing Handbook Fifth Edition”, CRC press,2006. 